\title{
Optimization-based Design of Plant-Friendly Input Signals for Model-on-Demand Estimation and Model Predictive Control
}

\author{
Hyunjin Lee and Daniel E. Rivera* \\ Control Systems Engineering Laboratory \\ Department of Chemical Engineering \\ Arizona State University, Tempe, Arizona 85287-6006 \\ Hans D. Mittelmann and Gautam Pendse \\ Dept. of Mathematics and Statistics \\ Arizona State University, Tempe, Arizona 85287
}

\begin{abstract}
The design of constrained, "plant-friendly" multisine input signals that optimize a geometric discrepancy criterion arising from Weyl's Theorem is examined in this paper. Such signals are meaningful for data-centric estimation and control methods, where uniform coverage of the output state-space contributes greatly to good performance. The optimization problem includes a search for both the Fourier coefficients and phases in the multisine signal, resulting in an uniformly distributed output signal that achieves a desirable balance between high and low gain directions, an important consideration when identifying strongly interactive multivariable systems. The solution involves very little user intervention and has significant benefits compared to multisine signals that minimize crest factor. The usefulness of this problem formulation is shown by applying it to a case study involving composition control of a binary distillation column.
\end{abstract}

\section{INTRODUCTION}

In recent years, there has been significant interest in data-centric dynamic modeling frameworks such as Just-inTime modeling [1], Model-on-Demand (MoD) estimation [2] and more recently, Direct Weight Optimization (DWO) [3]. The appeal of these modeling approaches is that they enable nonlinear estimation, while reducing the structural decisions made by the user and maintaining reliable numerical computations. The performance of these methods, however, is highly dependent upon the availability of quality, informative databases, and consequently, good experimental designs are an imperative. An important consideration in experimental design for this class of estimation methods is to achieve uniform coverage of regressors in the database. This paper examines the development of multisine input designs that meet this criterion while satisfying plant-friendliness constraints during identification testing. A plant-friendly identification test will produce data leading to a suitable model within an acceptable time period, while keeping the changes and variability in both input and output signals within user-defined constraints [4].

* To whom all correspondence should be addressed. Phone: (480) 9659476 Fax: (480) 965-0037; e-mail: daniel.rivera@asu.edu
The approach described in this paper for achieving uniformly distributed experimental designs for system identification relies on geometric discrepancy theory [5]. This is accomplished by minimizing a discrepancy function made up of trigonometric polynomials arising from Weyl's Theorem [6] that insure that the points are equidistant on a statespace. The optimization problem calls for minimizing this discrepancy function on the anticipated outputs of the system, subject to the requirements of an orthogonal "zippered" spectrum (used to enable multi-channel implementation) and while enforcing time-domain constraints on upper and lower limits, move sizes, and rates of change in either (or both) input and output signals. The optimization problem is solved using a state-of-the-art NLP solver (KNITRO 3.1) which uses an interior point trust region method and employs SQP techniques to solve the barrier subproblems.

The effectiveness of data resulting from an optimizationbased signal design using the proposed Weyl criteria is demonstrated in a binary high-purity distillation column case study by Weischedel and McAvoy [7], a demanding nonlinear and strongly interactive process application. A Model-on-Demand Model Predictive Control (MoD-MPC) algorithm as developed in [2], [8], [9] is evaluated using a Weyl-based data-centric experiment versus a multisine input design with equivalent harmonics but minimizing crest factor. Improvements in closed-loop performance are achieved in the Weyl-based data set, without the need to compromise plant-friendliness in the experimental design.

The paper is organized as follows: Section 2 briefly describes the MoD estimation framework and its corresponding MPC implementation. Section 3 presents the Weyl criterion that defines the geometric discrepancy objective, while Section 4 describes an optimization-based input design problem formulation for a 2 by 2 multivariable system. Section 5 shows the application of a design based on this formulation to both open-loop estimation and closed-loop control of the Weischedel-McAvoy column, while Section 6 contains a summary and conclusions. 


\section{Model-on-Demand Modeling Methodology}

Model-on-Demand is a data-centric, nonlinear black-box estimation method which enhances the classical local modeling problem. In $\mathrm{MoD}$, an adaptive bandwidth selector determines the size of data to be used for the local regression. The data is weighted using a kernel or weighting function. A local regression is performed using a linear or quadratic model to estimate the plant output at each time step; all observations are stored on a database and the models are built 'on demand' as the actual need arises. Local modeling techniques such as the MoD predictor use only small portions of data, relevant to the region of interest, to determine a model as needed. The variance/bias tradeoff inherent to all modeling is optimized locally by adapting the number of data and their relative weighting. As a consequence, the non-convex optimization problem associated with most global nonlinear modeling techniques is avoided. Moreover, the user is presented with fewer decisions regarding model structure and can use intuition developed from linear model identification as the basis for obtaining an accurate nonlinear model. MoD has been formulated into a comprehensive methodology for nonlinear identification and predictive control [2], [8], [9], [10], [11]. A Matlab-based tool for MoD estimation and control is available in the public domain [12].

\section{A. Model on Demand Estimation}

The MoD modeling formulation is described with a SISO process based on the approach of [2]. Consider a SISO process with nonlinear ARX structure, i.e.,

$$
y(k)=m(\varphi(k))+e(k), \quad k=1, \ldots, N
$$

where $m(\cdot)$ is an unknown nonlinear mapping and $e(k)$ is an error term modeled as random variables with zero mean and variance $\sigma_{k}^{2}$. The MoD predictor attempts to estimate output predictions based on a local neighborhood of the regressor space $\varphi(t)$. The regressor vector is of the form

$\varphi(t)=\left[\begin{array}{lllll}y(t-1) & \ldots & y\left(t-n_{a}\right) u\left(t-n_{k}\right) & \ldots & u\left(t-n_{b}-n_{k}\right)\end{array}\right]^{T}$

where $n_{a}, n_{b}$, and $n_{k}$ denote the number of previous outputs and inputs and the degree of delays in the model.

A local estimate $\hat{y}$ can be obtained from the solution of the weighted regression problem

$\hat{\beta}=\arg \min _{\hat{\beta}} \sum_{k=1}^{N} \ell(y(k)-\hat{m}(\varphi(k), \hat{\beta})) \times W\left(\frac{\|\varphi(k)-\varphi(t)\|_{M}}{h}\right)$

where $\ell(\cdot)$ is a quadratic norm function, $\|u\|_{M} \triangleq \sqrt{u^{T} M u}$ is a scaled distance function on the regressor space, $h$ is a bandwidth parameter controlling the size of the local neighborhood and $W(\cdot)$ is a window function (usually referred to as the kernel) assigning weights to each remote data point according to its distance from $\varphi(t)$ [11]. The window is typically a bell-shaped function with bounded support. These weights can be chosen to minimize the point-wise mean square error of the estimate. Assuming a local model structure

$$
m(\varphi(t), \beta)=\beta_{0}+\beta_{1}^{T}(\varphi(k)-\varphi(t))
$$

which is linear in the unknown parameters, an estimate can easily be computed using least squares methods. If $\beta_{0}$ and $\beta_{1}$ denote the minimizers of (3) using the model from (4), a one-step ahead prediction is given by

$$
\hat{y}(t)=m(\varphi(t), \hat{\beta})=\hat{\beta}_{0}
$$

Each local regression problem produces a single prediction $\hat{y}(t)$ corresponding to the current regression vector $\varphi(t)$. To obtain prediction at other locations in the regressor space, the weights change and new optimization problems must be solved. This stands in contrast to the global modeling approach where the model is fitted to data only once and then discarded. The bandwidth $h$ controls the neighborhood size and has a critical impact on the resulting estimate since it governs a trade-off between the bias and variance errors of the estimate. Traditional bandwidth selectors produce a single global bandwidth; in MoD estimation, a bandwidth is computed adaptively at each prediction.

\section{B. Model-on-Demand Model Predictive Control}

Model predictions from MoD estimates can be incorporated into a Model Predictive Control (MPC) framework as described in [2], [8] and [9]. The objective function is

$$
\begin{array}{rc}
\min _{\Delta u(k \mid k) \ldots \Delta u(k+m-1 \mid k)} & \sum_{\ell=1}^{p_{h}} Q_{e}(\ell)(\hat{y}(k+\ell \mid k)-r(k+\ell))^{2} \\
+ & \sum_{\ell=1}^{m_{h}} Q_{\Delta u}(\ell)(\Delta u(k+\ell-1 \mid k))^{2}
\end{array}
$$

subject to constraints on outputs $\left(0 \leq y(k) \leq y_{\max }\right)$, inputs $\left(0 \leq u(k) \leq u_{\max }\right)$, and their rate-of-change $\left(\Delta u_{\min } \leq \Delta u_{k} \leq\right.$ $\left.\Delta u_{\text {max }}\right)$. The weight functions $Q_{e}(\ell)$ and $Q_{\Delta u}(\ell)$ are adjusted to obtain desired levels of robustness and performance.

\section{Uniform Distribution OF INFINITE SEQUENCES - THE WEYL CRITERION}

Discrepancy theory deals with the distribution of points in space [5]. The Weyl criterion [6] gives the necessary and sufficient conditions for a sequence to be uniformly distributed in $[0,1)^{d}$, the $d$-dimensional unit interval. The criterion for a two-dimensional sequence can be summarized as follows:

Theorem. (H. Weyl, 1916) A sequence $\left\{y_{1}(k), y_{2}(k)\right\}$ is equidistributed in $[0,1)^{2}$ if and only if

$$
\lim _{N \rightarrow \infty} \frac{1}{N} \sum_{k=1}^{N} e^{2 \pi i\left(l_{1} y_{1}(k)+l_{2} y_{2}(k)\right)}=0
$$

$\forall$ sets of integers $l_{1}, l_{2}$ not both zero.

Decomposing (7) into real and imaginary parts we obtain that the sequence $\left\{y_{1}(k), y_{2}(k)\right\}$ is equidistributed in $[0,1)^{2}$ if and only if for all sets of integers $l_{1}, l_{2}$ (not both zero) the following conditions hold:

$$
\lim _{N \rightarrow \infty} \frac{1}{N} \sum_{k=1}^{N} \cos \left[2 \pi\left(l_{1} y_{1}(k)+l_{2} y_{2}(k)\right)\right]=0
$$


and

$$
\lim _{N \rightarrow \infty} \frac{1}{N} \sum_{k=1}^{N} \sin \left[2 \pi\left(l_{1} y_{1}(k)+l_{2} y_{2}(k)\right)\right]=0
$$

Weyl's criterion can readily be extended to higher dimensions, as needed by the requirements of the problem under consideration.

\section{Optimization Problem Formulation}

Our goal is to design an input signal that is uniformly distributed and as such has good directionality information in the output state space of the system; the latter goal is an important requirement when working with strongly interactive multivariable systems [13]. This assumes a priori knowledge of the plant model as either an equation or a computer program that is available to the optimizer. For simplicity of presentation we consider the input $u(k)$ and output $y(k)$ are vectors with two components; this corresponds to the dimensions of the Case Study presented in Section 5.

To meet the objectives of plant friendliness we need to impose bound constraints on both $u(k)$ and/or $y(k)$. Here, $z$ is one of $y_{1}, y_{2}, u_{1}, u_{2}$.

$$
|z(k)| \leq C_{z}, \quad k=0, \ldots, N_{s}-1
$$

The $C_{z}$ are user defined constants. Since a multisine is a periodic signal these need to be applied over one cycle length $N_{s}$. We would also like to have restrictions on the move size of $u(k)$ and $y(k)$, which is the difference between successive values in $u(k)$ and $y(k)$. We therefore impose the constraints,

$$
|z(k+1)-z(k)| \leq \Delta C_{z}, k=0, \ldots, N_{s}-1
$$

Again $\Delta C_{z}$ are user defined constants. The prediction of the plant output response must be determined from a model estimated from previous identification tests, or otherwise obtained a priori. These relationships are represented as

$$
\begin{aligned}
& y_{1}(k)=f_{1}\left(u_{1}, u_{2}, y_{1}, y_{2}\right), k=0, \ldots, N_{s}-1 \\
& y_{2}(k)=f_{2}\left(u_{1}, u_{2}, y_{1}, y_{2}\right), k=0, \ldots, N_{s}-1
\end{aligned}
$$

Here the arguments of $f_{1}$ and $f_{2}$ indicate the dependence of $y_{1}$ and $y_{2}$ on the values of the vectors $u_{1}, u_{2}, y_{1}$ and $y_{2}$. The inputs $u_{1}(k)$ and $u_{2}(k)$ are chosen per the multisine structure

$$
u_{j}(k)=\sum_{i=1}^{(m+1) n_{s}} \sqrt{2 \alpha_{i j}} \cos \left(\frac{2 \pi i}{N_{s}} k+\phi_{i j}\right)
$$

with Fourier coefficient bounds corresponding to a modified zippered spectrum as described below:

$$
\alpha_{i j}= \begin{cases}\geq 0, & i=j,(m+1)+j, \cdots,(m+1)\left(n_{s}-1\right)+j \\ \geq 0, & i=m+1,2(m+1), \cdots, n_{s}(m+1) \\ =0, & \text { for all other } i \text { up to }(m+1) n_{s}\end{cases}
$$

The goal is to uniformly distribute the points $\left(y_{1}(k), y_{2}(k)\right)$ in the output state space region $\left[-C_{y_{1}}, C_{y_{1}}\right) \times\left[-C_{y_{2}}, C_{y_{2}}\right)$. We wish to use the Weyl Criterion described in the previous section to achieve this uniform distribution. Since the Weyl Criterion deals with uniform distributions in $[0,1)^{2}$, we introduce a change of variables:

$$
\hat{y}_{1}(k)=\frac{y_{1}(k)+C_{y_{1}}}{2 C_{y_{1}}}, \quad \hat{y}_{2}(k)=\frac{y_{2}(k)+C_{y_{2}}}{2 C_{y_{2}}}
$$

Since we only have a finite number of points in the sequences, we choose an integer $L$ and form the set $S$ as follows:

$$
S=\{x: x \in Z \text { and }|x| \leq L\}
$$

where $Z$ is the set of all integers and $W$ corresponds to

$$
W=\left\{\left(l_{1}, l_{2}\right): l_{1} \in S, l_{2} \in S \text { and }\left(l_{1}, l_{2}\right) \neq(0,0)\right\}
$$

We then try to minimize the sum in equations (8) and (9) for all elements of the set $W$. The optimization is carried out to estimate the amplitudes and phases $\alpha_{i 1}, \alpha_{i 2}, \phi_{i 1}, \phi_{i 2}, i=$ $1, \ldots,(m+1) n_{s}$ of the $m=2$ multisine input channels. The complete problem statement is as follows:

$$
\min _{\alpha_{i 1}, \alpha_{i 2}, \phi_{i 1}, \phi_{i 2}} t
$$

s.t.

$$
\begin{gathered}
\sum_{k=0}^{N_{s}-1} \cos \left[2 \pi\left(l_{1} \hat{y}_{1}(k)+l_{2} \hat{y}_{2}(k)\right)\right] \leq t, \forall\left(l_{1}, l_{2}\right) \in W \\
\sum_{k=0}^{N_{s}-1} \sin \left[2 \pi\left(l_{1} \hat{y}_{1}(k)+l_{2} \hat{y}_{2}(k)\right)\right] \leq t, \forall\left(l_{1}, l_{2}\right) \in W \\
t \geq \varepsilon
\end{gathered}
$$

as well as subject to constraints per Equations (10)-(15). The lower bound constraint on $t$ is imposed to promote faster convergence. $\varepsilon$ is chosen to be some small positive constant.

[14] and [15] paper present experiments that show the influence of design variables $L$ and $\varepsilon$ on the distribution of points in the output state space. In these experiments it can be seen that by increasing $L$, the uniformity in the output state space distribution improves dramatically. Decreasing the value of $\varepsilon$ tends to keep the various clusters of points in more or less the same position, but leads to a redistribution of points within the same cluster. Given our experience, there is not much to gain by decreasing $\varepsilon$ beyond a certain limit; it is, however, much more advantageous to increase $L$ instead.

The constrained optimization problems described in this paper were solved by programming them in the modelling language AMPL which has built in automatic differentiation up to second order derivatives. The Weyl equations are continuously differentiable and so the optimizer can make direct use of second derivative information. The optimizer used was KNITRO developed by Byrd and co-workers [16]. KNITRO is an interior point trust region SQP solver and is suitable for solving both large and small problems.

\section{Case Study: Nonlinear High-Purity Distillation PROCESS}

\section{A. Input Signal Design and Comparison to Minimum Crest Factor Approaches}

A challenging multivariable process system that benefits from judiciously applied system identification techniques is high purity distillation. The methanol-ethanol distillation column model developed by Weischedel and McAvoy [7] is commonly used as a benchmark problem for this type of application [17]. To address the demands of highly interactive 


\begin{tabular}{|c|c|c|c|c|c|c|}
\hline Type & Signal $(x)$ & $\mathrm{CF}(x)$ & PIPS(\%) & $\max \Delta x$ & $\max x$ & $\min x$ \\
\hline \multirow{4}{*}{ min CF $(u)$ design; standard zippered spectrum } & $u_{1}$ & 1.21 & 82.43 & 0.0025 & 0.0020 & -0.0020 \\
\hline & $u_{2}$ & 1.22 & 81.77 & 0.0026 & 0.0020 & -0.0020 \\
\hline & $y_{1}$ & 2.48 & 48.84 & 0.0037 & 0.0325 & -0.0211 \\
\hline & $y_{2}$ & 2.19 & 46.12 & 0.0031 & 0.0199 & -0.0204 \\
\hline \multirow{4}{*}{$\begin{array}{l}\text { min } \mathrm{CF}(y) \text { design; modified zippered spectrum } \\
\text { using NARX model prediction }|\Delta u| \leq 0.01 \text {, } \\
|\Delta y| \leq 0.008 \&|y| \leq 0.0085\end{array}$} & $u_{1}$ & 3.74 & 31.51 & 0.0100 & 0.0365 & -0.0254 \\
\hline & $u_{2}$ & 3.25 & 34.37 & 0.0100 & 0.0316 & -0.0250 \\
\hline & $y_{1}$ & 1.30 & 77.45 & 0.0051 & 0.0088 & -0.0086 \\
\hline & $y_{2}$ & 1.31 & 77.01 & 0.0082 & 0.0087 & -0.0086 \\
\hline \multirow{4}{*}{$\begin{array}{l}\text { Data-centric Weyl design using NARX model via } \\
\text { a modified zippered spectrum subject to } \\
|\Delta u| \leq 0.01,|\Delta y| \leq 0.08 \&|y| \leq 0.0085\end{array}$} & $u_{1}$ & 2.78 & 37.52 & 0.0079 & 0.0292 & -0.0268 \\
\hline & $u_{2}$ & 2.50 & 41.28 & 0.0076 & 0.0240 & -0.0225 \\
\hline & $y_{1}$ & 1.79 & 56.54 & 0.0062 & 0.0084 & -0.0082 \\
\hline & $y_{2}$ & 1.76 & 57.13 & 0.0053 & 0.0082 & -0.0083 \\
\hline
\end{tabular}

TABLE I

DESCRIPTION AND RESULTS SUMMARY FOR MULTISINE SIGNALS DESIGNED FOR THE WEISCHEDEL-MCAVOY DISTILLATION COLUMN CASE STUDY.

systems, one approach is to modify the standard multisine signal to contain correlated harmonics with high levels of power, which improve the low gain-direction content in the data and promote better coverage of the output state-space. Such an approach is presented in [18] and [19], who apply an optimization approach that minimizes crest factor $(C F)$, the ratio of the $\ell_{\infty}$ (or Chebyshev) norm and the $\ell_{2}$-norm of a signal $x$ [20]. A low crest factor indicates that most of the elements in the sequence are distributed near their extremum values. Design parameters for the WeischedelMcAvoy problem determined on the basis of the guidelines per [18] using dominant time constant estimates $\left(\tau_{d o m}^{L}=5\right.$ and $\tau_{d o m}^{H}=20 \mathrm{~min}$ ) and user choices of $\delta=0, \alpha_{s}=2$, and $\beta_{s}=3$, lead to parameter settings of $T=2$ minutes, $n_{s}=189$, and $N_{s}=378$. Constraints applied to the problem and salient characteristics of the signals considered (min $\mathrm{CF}(u)$, min $\mathrm{CF}(y)$, and Weyl data-centric) are summarized in Table I. The value of the amplification factor for the correlated harmonics $\gamma=15$ was chosen for a min $\operatorname{CF}(y)$ signal with modified spectrum; the resulting input spectrum for this signal is shown in Figure 1a. The output state-space plot is shown in Figure 2a.

A significant benefit of an optimization-based problem formulation for input signal design is that nonlinear model forms can be readily incorporated in the design procedure, which results in an improved ability to both meet plantfriendliness requirements as well as address the directionality and uniform distribution requirements in the output for demanding applications. A polynomial Nonlinear AutoRegressive with eXternal (NARX) input model with structure as proposed in [17]

$$
\begin{aligned}
y(k)= & \theta^{(0)}+\sum_{i=1}^{n_{y}} \theta_{i}^{(1)} y(k-i)+\sum_{i=\rho}^{n_{u}} \theta_{i}^{(2)} u(k-i)+ \\
& +\sum_{i=1}^{n_{y}} \sum_{j=1}^{i} \theta_{(i, j)}^{(3)} y(k-i) y(k-j) \\
& +\sum_{i=\rho}^{n_{u}} \sum_{j=\rho}^{i} \theta_{(i, j)}^{(4)} u(k-i) u(k-j) \\
& +\sum_{i=1}^{n_{y}} \sum_{j=\rho}^{n_{u}} \theta_{(i, j)}^{(5)} y(k-i) u(k-j)+\ldots
\end{aligned}
$$

was obtained for the Weischedel-McAvoy column and used to generate output predictions for the optimizer in both the min $\mathrm{CF}(y)$ and Weyl-based signal design scenarios.

The benefits of the Weyl-based formulation over the minimum crest factor signal design in producing a uniform distribution in the output state-space of the data can be clearly seen by contrasting Figures $2 \mathrm{a}$ and $2 \mathrm{~b}$ : the use of the Weyl-based criterion results in a much more uniformly distributed coverage of the state-space, and a much better suited dataset for data-centric estimation purposes. The uniform distribution of the output within the bounds specified in the problem results in a natural balance between the high and low gain information content in the data. From Table I one does notice, however, that the improvement in output state space uniformity is obtained at the cost of higher crest factor, which consequently reduces the signal-to-noise ratio of the data in a noisy data setting. As a result there is an inherent tradeoff between these objectives that needs to be recognized. One way of addressing this issue in practical input design is to include maximum crest factor bounds as inequality constraints within the Weyl problem formulation; these can be readily incorporated in the numerical optimization framework described in this paper.

An important difference between these signal designs is observed in the input spectra (Figure 1). In the min CF (y) case, only the phases and a subset of the Fourier coefficients in the high frequency range of the multisine signal are chosen by the optimizer, while for the Weyl-based design, the optimization problem includes a search for all Fourier coefficients and phases, including those corresponding to the correlated harmonics; this can be seen in Figure 1b. Not only do these extra degrees of freedom in the optimizer contribute to the improved performance, they reduce the number of decisions made a priori by the user, leading to a more practical design procedure.

\section{B. Application to Model-on-Demand Estimation and Predic- tive Control}

First, open-loop Model-on-Demand (MoD) estimation is evaluated for the Weischedel-McAvoy distillation column using for estimation purposes the data arising from the signal designs summarized in Table I. For validation purposes, a different data-centric signal with lower magnitude bounds was considered, which is shown in [19]. For all cases, an 


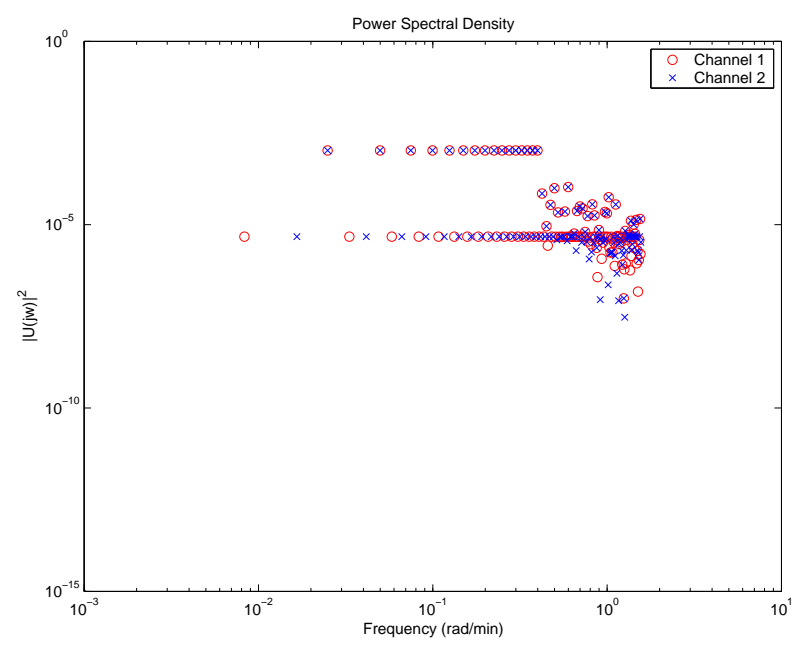

(a) $\min \mathrm{CF}(y)$

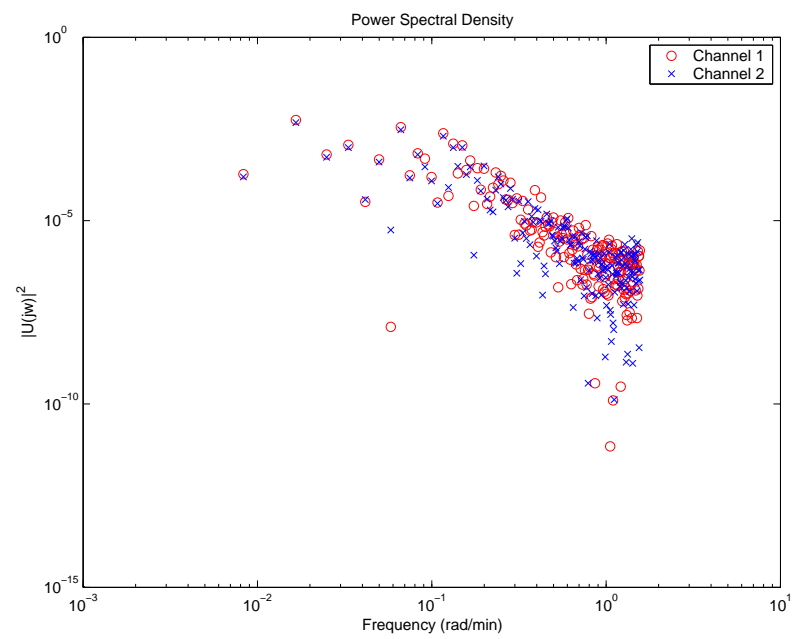

(b) Weyl-based Approach

Fig. 1. Power spectral densities for input signals for the WeischedelMcAvoy distillation column: $\min \mathrm{CF}(y)$ (a) versus Weyl-based design (b)

implicit Nonlinear ARX (NARX) structure with $\left[n_{a}=1\right.$, $\left.n_{b}=1, n_{k}=1\right]$ is used in the MoD estimator. A local polynomial order per (4) and limits on the database size $k_{\text {min }}=20$ and $k_{\text {max }}=756$ serve as additional parameters [10].

Analysis of the results shows that data resulting from the min $\mathrm{CF}(u)$ signal based on the standard zippered spectrum provides the worst results of all three cases considered. Because the input-output data resulting from this signal lacks information content in the low gain direction, it results in the poorest prediction on the validation data set (Table II). For data resulting from the $\min \mathrm{CF}(y)$ signal with modified spectrum, most of the output sequence is located near its minimum and maximum values, but its corresponding MoD model still seems to produce reasonable predictions in an open-loop sense with a percent unexplained variance of $0.5 \%$ (Table II). Nonetheless, the MoD model estimated from the Weyl-based approach, because of the even state-space distribution, results in the most accurate predictions of all

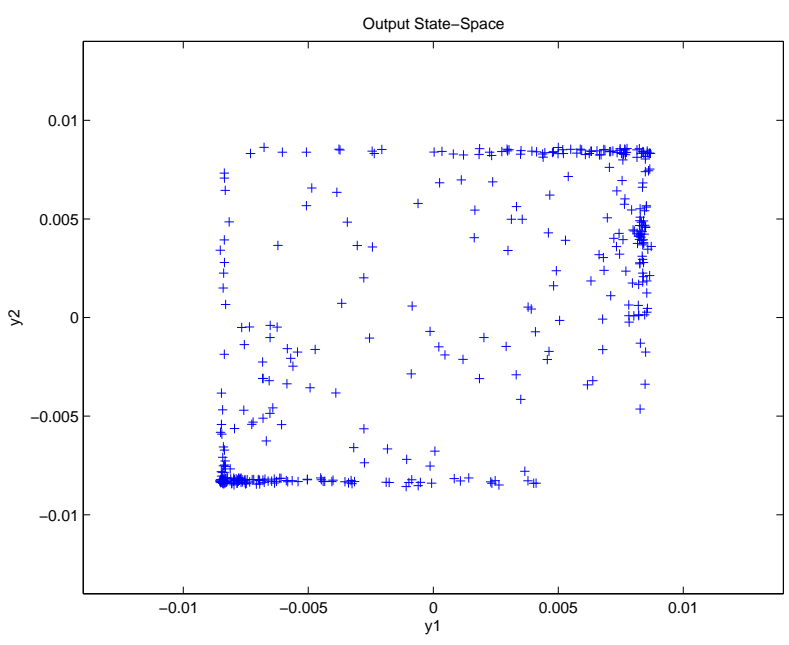

(a) $\min \mathrm{CF}(y)$

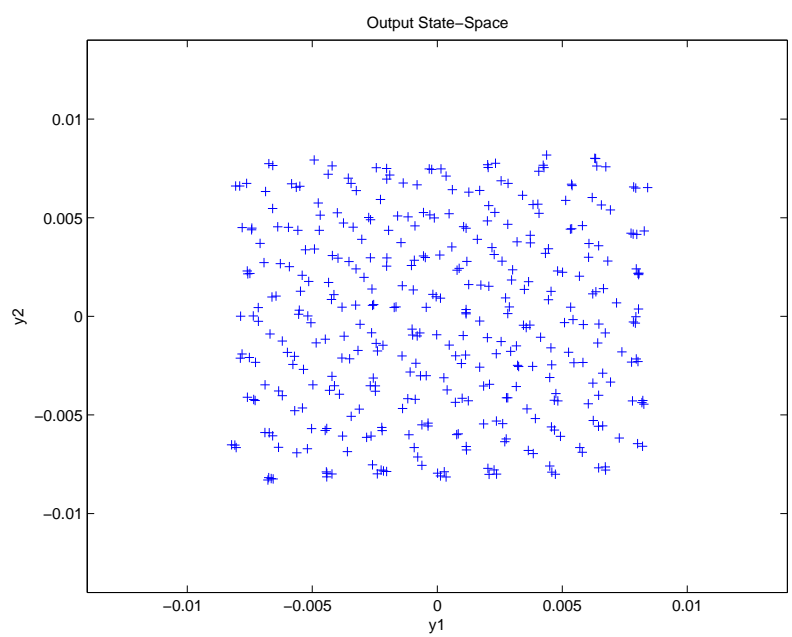

(b) Weyl-based Approach

Fig. 2. Output state-space plots for Weischedel-McAvoy distillation column: $\min \mathrm{CF}(y)$ (a) versus Weyl-based design (b)

signals considered, with a percent unexplained variance close to $0.09 \%$ (Table II).

The min CF(y) and Weyl-based MoD models are subsequently evaluated in a closed-loop setting using the MoDMPC Toolbox [12]. Results for the min CF(u) MoD model are not shown because a stable controller response could not be obtained. In both cases the tuning parameters are $p_{h}=35, m_{h}=15, Q_{e}=\left[\begin{array}{lll}1 & 0 ; 0 & 1\end{array}\right]$ and $Q_{\Delta u}=\left[\begin{array}{lll}7 & 0 ; 0 & 0\end{array}\right]$. A series of setpoint changes that represent challenges to controller performance for a highly interactive plant such as high-purity distillation are shown in Figure 3. While stable responses are obtained in both cases, the MoD-MPC controller relying on the Weyl-based data shows faster settling time, less overshoot, and less interaction than the one resulting from the min $\mathrm{CF}(y)$ MoD model; these desirable performance features of the Weyl-based MoD-MPC controller point to the effectiveness of this class of signals for closed-loop control purposes in a demanding process application. 


\begin{tabular}{lrrr}
\hline Type & Signal $(y)$ & RMS & Eval $(\%)$ \\
\hline min CF $(u)$ design; standard & $y_{1}$ & 1.5246 & 1011003.038 \\
\cline { 2 - 4 } zippered spectrum & $y_{2}$ & 3.2170 & 1803825.964 \\
\hline min CF $(y)$ design; modified & $y_{1}$ & 0.0002316 & 0.422 \\
\cline { 2 - 4 } zippered spectrum & $y_{2}$ & 0.0002550 & 0.531 \\
\hline \multirow{2}{*}{ Data-centric Weyl design } & $y_{1}$ & 0.0001018 & 0.090 \\
\cline { 2 - 4 } & $y_{2}$ & 0.0001070 & 0.093 \\
\hline
\end{tabular}

TABLE II

SUMMARY OF OPEN-LOOP CROSSVALIDATION RESULTS FOR THE WEISCHEDEL-MCAVOY DISTILLATION COLUMN CASE STUDY.
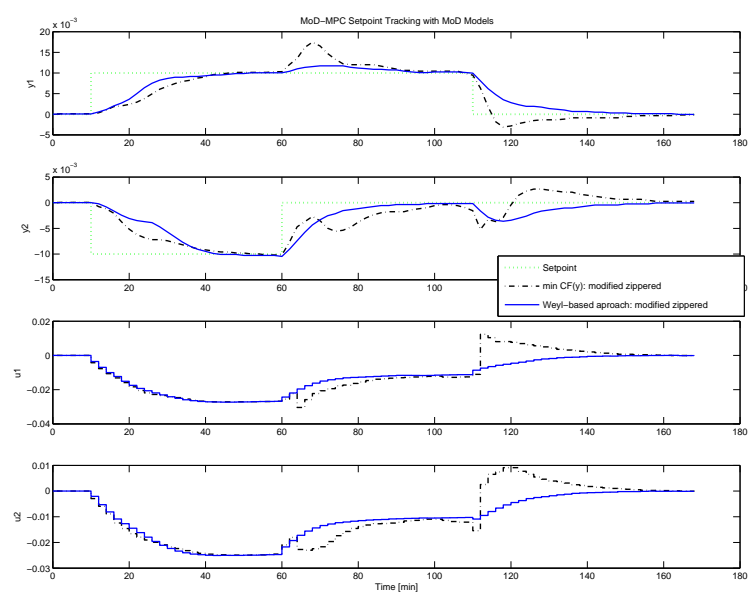

Fig. 3. MoD-MPC closed-loop setpoint tracking test on the WeischedelMcAvoy distillation column using MoD Models from the min CF (y) signal (dashed) and Weyl-based (solid) signal designs. Controller parameters are $p_{h}=35, m_{h}=15, Q_{e}=\left[\begin{array}{llll}1 & 0 ; 0 & 1\end{array}\right]$ and $Q_{\Delta u}=\left[\begin{array}{lll}7 & 0 ; & 0\end{array}\right]$

\section{SUMmARY AND CONCLUSIONS}

This paper has described a novel constrained optimizationbased formulation of the multisine input signal problem, meaningful for data-centric estimation and control schemes such as those based on the concept of Model-on-Demand. The objective function is based on the Weyl criterion, which seeks to minimize the geometric discrepancy of the output in the state-space. This contrasts earlier work on optimizationbased plant-friendly approaches to the problem based on minimizing crest factor. An problem formulation for a 2 by 2 system was presented and evaluated on a case study centered on a high purity binary distillation column, a challenging nonlinear and strongly interactive multivariable process system. The improvements achieved by the Weyl-based solution, both from an open-loop estimation perspective and from the standpoint of closed-loop control using a MoD-based MPC control algorithm are demonstrated. The power of the proposed framework lies not only in its performance but also in its flexibility, allowing the user to incorporate both linear and nonlinear models for output prediction, time-domain constraints, and information and control-theoretic frequency domain requirements. The use of state-of-the-art interiorpoint optimization methods enables the efficient solution of these nonlinear and nonconvex optimization problems.

\section{ACKNOWLEDGMENTS}

Funding for this work was provided by the American Chemical Society-Petroleum Research Fund through grant number PRF-37610-AC9. We also wish to thank Dr. Martin W. Braun, currently at Intel Corp., for introducing us to the concept of geometric discrepancy.

\section{REFERENCES}

[1] G. Cybenko, "Just-in-time learning and estimation," in Identification, Adaptation, Learning, ser. NATO ASI, S. Bittani and G. Picci, Eds. Springer, 1996, pp. 423-434.

[2] A. Stenman, "Model on demand: Algorithms, analysis and applications," Ph.D. dissertation, Linköping University, Linköping University, Sweden, 1999.

[3] J. Roll, A. Nazin, and L. Ljung, "Nonlinear system identification via direct weight optimization," Automatica, vol. 41, pp. 475-490, 2005.

[4] D. Rivera, H. Lee, M. Braun, and H. Mittelmann, "Plant-friendly system identification: a challenge for the process industries," in 13th IFAC Symposium on System Identification (SYSID 2003), Rotterdam, Netherlands, 2003, pp. 917-922.

[5] J. Matousek, Geometric Discrepancy: An Illustrated Guide. Berlin: Springler-Verlag, 1999.

[6] H. Weyl, "Über die gleichverteilung von zahlen mod eins," Math. Ann., vol. 77, pp. 313-352, 1916.

[7] K. Weischedel and T. McAvoy, "Feasibility of decoupling in conventionally controlled distillation columns," Ind. Eng. Chem. Fund., vol. 19 , p. 379,1980

[8] M. Braun, D. Rivera, and A. Stenman, "Model-On-Demand Model Predictive Control for nonlinear process systems," 2000 AIChE Annual Meeting, vol. 256h, pp. $1-40,2000$.

[9] M. W. Braun, "Model-on-demand nonlinear estimation and model predictive control: Novel methodologies for process control and supply chain management," Ph.D. dissertation, Dept. of Chemical and Materials Engineering, Arizona State University, 2001.

[10] M. Braun, D. Rivera, and A. Stenman, "A Model-on-Demand identification methodology for nonlinear process systems," Int. J. Control, vol. 74, no. 18, pp. 1708-1717, 2001.

[11] M. Braun, R. Ortiz-Mojica, and D. Rivera, "Application of minimum crest factor multisinusoidal signals for "plant-friendly" identification of nonlinear process systems," Control Engineering Practice, vol. 10, p. $301,2002$.

[12] M. Braun, A. Stenman, and D. Rivera, "Model-on-Demand Model Predictive Control Toolbox," http://www.fulton.asu.edu/ csel/Software.htm, 2002.

[13] S. Skogestad and I. Postlethwaite, Multivariable feedback control: analysis and design, 2nd ed. Hoboken, NJ: Wiley, 2005.

[14] G. Pendse, "Optimization-based formulations using the Weyl criterion for input signal design in system identification," Master's thesis, Arizona State University, Tempe, AZ, U.S.A, May 2004.

[15] D. Rivera, H. Lee, H. Mittelmann, and G. Pendse, "Optimization-based design of plant-friendly input signals using geometric discrepancy criteria," in 14th IFAC Symposium on System Identification (SYSID 2006), Newcastle, Australia, 2006, pp. 1133 - 1138.

[16] R. Byrd, M. Hribar, and J. Nocedal, "An interior point method for large scale nonlinear programming," SIAM J. Optim., vol. 9, pp. 877-900, 1999.

[17] G. R. Sriniwas, Y. Arkun, I.-L. Chien, and B. Ogunnaike, "Nonlinear identification and control of a high-purity distillation column: a case study," J. Proc. Cont., vol. 5, p. 149, 1995.

[18] H. Lee, D. Rivera, and H. Mittelmann, "Constrained minimum crest factor multisine signals for plant-friendly identification of highly interactive systems," in 13th IFAC Symp. on System Identification, Rotterdam, 2003, pp. 947-952.

[19] H. Lee, "A plant-friendly multivariable system identification framework based on identification test monitoring," Ph.D. dissertation, Dept. of Chemical Engineering, Arizona State University, Tempe, AZ, U.S.A, 2006

[20] P. Guillaume, J. Schoukens, R. Pintelon, and I. Kollár, "Crest-factor minimization using nonlinear Chebyshev approximation methods," IEEE Trans. on Inst. and Meas., vol. 40, no. 6, pp. 982-989, 1991. 